\title{
La féminité et le mouvement
}

\author{
Houriya Abdelouahed
}

«Dans l'étreinte amoureuse de la traduction, écrit Paul Valéry, les langues comme les amants, étreignent ce qu'elles ignorent». Toutefois, c'est sur le fond de ce qui demeure, par elles, ignoré que se fait la rencontre. Et la traduction, note Walter Benjamin, a pour dessein d'exprimer le rapport le plus intime entre les langues, de dire leur parenté ${ }^{1}$ à condition toutefois de ne pas confondre parenté et ressemblance. La traduction loin d'abolir la différence entre les langues, se fait en elle ${ }^{2}$. Et l'étreinte amoureuse se nourrit de cette différence entre les langues, de la tension de l'écart puisque la traduction est une manière « de se mesurer à ce qui rend les langues étrangères l'une à l'autre ${ }^{3}$. Ceci étant, la trahison est là également comme une toile de fond puisque la traduction ne parvient pas à révéler ou à restituer ce rapport caché lui-même. Elle ne peut que le représenter " en l'actualisant dans son germe ou dans son intensité » ${ }^{4}$. La tâche du traducteur consiste alors à trouver, dit encore Walter Benjamin, " dans la langue où est traduit l'original, cette visée intentionnelle qui éveille en elle l'écho de l'original $»^{5}$.

Cette visée intentionnelle est ce qui anime ma réflexion aujourd'hui sur la féminité. Suivre les sentiers d'un texte et le rythme de sa langue-dans une tentative de me rapprocher de l'écho originel dont les différentes traductions se sont écartées - permet de mesurer les enjeux d'un tel écart.

1. Benjamin W., Mythe et violence, I, Denoël, 1971, p. 264.

2. Gomez Mango E., La Mort enfant, Gallimard, Coll. «Tracé », 2003, p. 73.

3. W. Benjamin, op. cit., p. 267.

4. Op. cit., p. 264

5. Op. cit., p. 269. 
Le verset 31 de la sourate 24 (Cor. 24 :31) fut traduit de la façon suivante :

- «Commande aux fidèles de contenir la licence de leurs regards et $d$ 'être chastes. Ordonne aux femmes de baisser les yeux, de conserver leur pureté» (trad. M. Savary)

- «Dis aux croyants de baisser les yeux et de contenir leur sexe. Ce sera de leur part plus net. Dieu est de leur pratiques informé. Dis aux croyantes de baisser les yeux et de contenir leur sexe.» (J. Berque)

- «Dis aux adhérents de baisser leurs regards, de préserver leur nudité. Dis aux adhérentes de baisser leurs regards, de préserver leur nudité.» (A. Chouraqui)

- «Dis aux croyants de baisser les yeux, d'être chastes. Dis aux croyantes de baisser les yeux, d'être chastes.» (J. Grosjean)

- «Dis aux croyants de tenir leurs yeux pudiquement baissés et de préserver leur sexe. De même aux croyantes de baisser non moins pudiquement leurs regards et de protéger leur vertu.» (Sadok Mazich)

- «Dis aux croyants de baisser leurs regards et de maîtriser leurs passions sexuelles. C'est plus pur pour eux. Et dis aux croyantes de baisser leur regard et de maîtriser leurs passions sexuelles.»(Gilles Valois)

- «Commandez aux croyants de baisser leurs regards et $d$ 'être chastes. Ils en seront plus purs. Commande aux femmes qui croient de baisser leurs yeux et $d$ 'être plus chastes.» (Kasimirski)

- « Dis aux croyantes de baisser leur regards, d'être chastes. Dis aux croyantes de baisser leur regards, d'être chastes. » (D. Masson)

- «Dis aux croyants de baisser les yeux et de garder avec soins leurs fissures. Cela est plus pur pour eux. Dis aux croyantes de baisser les yeux et de garder avec soins leurs fissures.» (René Khawam)

Le terme arabe qui fut traduit par nudité, maîtrise des passions sexuelles, vertu, chasteté ${ }^{6}$, fissures... est al-farj, le sexe. Tout verset coranique stipulant la préservation du sexe, écrit le grand commentateur Tabari (IX ${ }^{\mathrm{e}}$ siècle), concerne sa préservation ou sa protection contre ce qui est illicite (zinâ, adultère ; luwata, pédérastie) sauf ce verset. Ici, le Coran interdit la vue des furûj (pluriel de farj). La première phrase, continue le commentateur, se trouve indissolublement liée à la seconde. Ainsi se trouve tissé un lien entre la vue (et non le regard) et le génital ${ }^{7}$.

Lisân al- 'arab, encyclopédie philologique du XIII' siècle nous apprend qu'alfarj est la fente, la fissure, la crevasse, espace compris entre les deux. Furûj sont les parties honteuses tant de l'homme que de la femme tant de devant que de derrière ${ }^{8}$. Faraja: fendre, pourfendre, ouvrir (une porte par exemple), entrou-

6. Outre la lecture chrétienne de ce verset (chasteté, vertu...), nous voyons également l'influence du discours sociologique sur le travail de traduction. Ainsi dans celle de Sadok Mazich, du côté des hommes, il y a le sexe et du côté des femmes, la vertu.

7. Tabarî, Jâmi' al-bayân 'an tafsîr âyi al-Qur'ân, Beyrouth (Liban), éd. Dâr al-fikr, 1988.

8. Définition qui va dans le sens de ce que nous nommons une «confusion orificielle». 
vrir, dissiper (les soucis), disperser, écarter, espacer. Furûj (adjectif) se dit également d'une personne ne pouvant garder un secret. Farîj: fente, fissure, espace interstice entre les doigts. Furûj: qui a les deux bouts très distancés. Tifrija, outre les sens déjà indiqués, désigne le peigne à démêler.

Ce farj-ouverture interroge à plus d'un titre. Et il revient à Fethi Benslama le mérite d'avoir attiré l'attention sur l'existence au sein de la langue arabe d'un seul terme pour désigner aussi bien le sexe de l'homme que celui de la femme. Mais aujourd'hui, note-t-il, les Arabes l'ont perdu puisqu'il désigne désormais le seul organe sexuel de la femme ${ }^{9}$. Fethi Benslama interroge notamment une lecture d'Abdel-kebir Khatibi qui écrit : «Afin de mieux situer la position de la sexualité en islam, il convient de considérer la différence sexuelle comme secondaire par rapport à la différence entre croyance et mécréance ${ }^{10}$. Faisons remarquer que cette lecture de Khatibi est lourde de conséquences. En omettant le «Nous vous avons créés à partir d'un mâle et d'une femelle » (Cor. 49-13), ou encore «Le masculin n'est pas comme le féminin » (Cor. 3 :36), A. Khatibi oublie que le Texte œuvre pour une pensée de la différence et inscrit la différence sexuelle au cœur même du pensable. Fethi Benslama continue : le farj comme «représentation d'un pouvoir-impouvoir qui se signifie en langue arabe par le manque, on comprend vite qu'il s'agit de ce qu'on désigne en psychanalyse par le terme de phallus ». Et «tout ce qui est aliénation fondamentale de l'homme dans l'islam serait donc pensée à partir du sexe absolu, ou selon une formulation psychanalytique, à partir du primat phallique ${ }^{11}$.

Notre lecture est différente.

Nous pouvons noter dans un premier temps à l'instar de Françoise Héritier que la différence des sexes est une donnée naturelle et que de son observation découlent des notions abstraites dont le prototype est l'opposition identique/différent sur lesquelles se moulent toutes les oppositions conceptuelles ${ }^{12}$. Disons, ensuite, que cette différence qui est au cœur même de la pensée, source de la pensée, embarrasse la théorie psychanalytique. Comme le note Claude Levesque ${ }^{13}$, l'embarras freudien devant «l'énigme de la féminité » est en fait un malaise devant la différence des sexes. Ainsi la passion freudienne du dévoilement ${ }^{14}$ cède-t-elle la place à des théories héritées de l'Antiquité. De la fantasmatique théorique sur un excès du féminin engendrant le monstrueux jusqu'à cette fameuse phrase de Napoléon reprise par le père de la psychanalyse, «L'anatomie c'est le destin », on remarque que les bonnes ou les mauvaises volontés viennent ainsi se heurter au roc du biologique. Et le malaise freudien devant ce

9. Benslama F., «Le sexe absolu », in Cahiers intersignes, $\mathrm{n}^{\circ}$ 2, printemps 1991, p. 107.

10. Cité par Benslaman F. op. cit., p. 113.

11. Benslama F., «Le sexe absolu », p. 117 et p. 118.

12. Cf. Héritier F., Masculin/féminin, éd. Odile Jacob, 1996, p. 26.

13. Levesque C., Par-delà le masculin et le féminin, Aubier, 2002.

14. Cette façon qu'avait Freud de jeter de la lumière sur des zones inexplorées ou interdites, de dire par exemple que les gestes les plus sacrés s'ancrent dans le pulsionnel et le fantasmatique. 
qu'il nomme l'énigme de la féminité s'accentue dans les textes tardifs ${ }^{15}$ œuvrant pour une version masculine de la différence sexuelle excluant ainsi le garçon de toute historicité ${ }^{16}$. Autant la petite fille est posée par rapport à un devenir (le devenir féminin de la petite fille) autant le garçon ne s'inscrit dans aucun devenir. Tout est réfléchi en fonction de l'organe emblématique mettant la fille du côté d'un masculin atrophié. Ainsi, le stade phallique - semblable pour les deux sexes - reste entièrement voué au membre viril. Le clitoris est un petit pénis et le vagin comme zone organisatrice de la sexualité féminine adulte serait sans enracinement dans l'infantile. Positions dont s'écarteront Karl Abraham, Melanie Klein, Ernst Jones...

La psychanalyse a fait sienne une version masculine de la différence sexuelle. Lacan dira plus tard : "L'imaginaire ne fournit qu'une absence là où il y a ailleurs un symbole très valent ${ }^{17}$ et «L'organe privilégié de la puissance (...) est ce que l'homme a sous la main ». Cette «vision» d'un organe emblématique fondant le privilège de l'un et la domination de l'autre imprègne indéniablement la traduction de Sadok Masich qui s'écartant d'une pensée chrétienne de la chasteté et la pureté, n'en demeure pas moins dans les confins d'un discours sociologico-moral demandant aux hommes de conserver leur sexe et aux femmes de protéger leur vertu.

Dans Généalogie du masculin et Le Paradigme féminin, Monique Schneider va, dans sa lecture et, comme elle le dit elle-même, au-delà des contradictions internes et des fissures théoriques qui traversent l'œuvre freudienne quant à cette question : père-mère/spiritualité-sensorialité (de cette fameuse lettre où le père est défini dans sa fonction plutôt contraceptrive ${ }^{18}$ jusqu'à L'Homme Moïse passant par le Malaise où la femme est définie comme inhibitrice du processus de civilisation) pour s'intéresser plus particulièrement aux phénomènes d'ancrage pulsionnel qui se jouent à même l'écriture ${ }^{19}$. L'auteur dénonce une «dérision de la verticalité comme paradigme du masculin » ${ }^{20}$; à savoir une Gestalt «impliquant un rapport spécifique à la spatialité : pierre dressée, érection, homme devenu paradigmatique de la verticalité » ${ }^{21}$, laquelle verticalité se trouve nourrie par cette homophonie dans la langue allemande-sur laquelle Jacques André attire l'attention - entre nämlich (même-identique) et nämlichen (masculin-viril). Laquelle homophonie est absente de la langue arabe car le même se dit nafsuhu, et le masculin se dit dhakar ou mudakkar.

15. Levesque C. écrit : «On le (Freud) surprend à pratiquer, dès que le sujet s'annonce, une sorte d'hésitation embarrassée, d'ignorance avouée ou d'agnosticisme », op. cit., p. 26.

16. Schneider M., Généalogie du masculin, Aubier, 2000 et Le Paradigme féminin, Aubier, 2004.

17. Lacan J., Le Séminaire, Livre III, Les psychoses, Seuil, 1981, p. 198.

18. Cf. Schur M., La Mort dans la vie de Freud, Gallimard, 1975, pp. 245-246.

19. Schneider M., La Paradigme féminin, p. 17.

20. op. cit., p. 115.

21. op. cit., p. 288. 
Dans Aux origines féminines de la sexualité, Jacques André développe l'idée d'une féminité originaire refoulée. La théorie de la séduction généralisée fait que l'enfant (fille ou garçon) est un enfant séduit. L'enfant séduit est un enfant orificiel $^{22}$. Il est pénétré par effraction, l'effraction de la passion adulte dans le monde de la tendresse de l'enfant. Cette effraction séductrice originaire de l'adulte fait de l'enfant un enfant orificiel. Dire qu'il y a un primat du phallus, nous fait oublier le noyau inconscient au profit d' « une organisation » ou d' « un rapport d'ordonnancement», écrit Jacques André. «En tant que fonction distributive des représentations », poursuit-il, le primat du phallus ne peut être inconscient $^{23}$. Ainsi la thèse freudienne s'avère une «manifestation du fantasme qui la sous-tend: le primat du phallus $\gg .{ }^{24}$

Afin d'arracher la figure de l'homme à une vision purement spirituelle, Monique Schneider le re-met en contact avec le sensible. Seulement l'hybridité, note Claude Levesque, ne parvient pas à rompre avec deux essences parfaitement identifiables et antithétiques. «Le fait d'intervertir les termes d'une opposition n'annule en rien la présence d'un terme sur l'autre. Contre toute attente, le schéma dichotomique auquel cherche à échapper l'auteure finit par contaminer la Généalogie, par imposer sa loi. En s'opposant à l'opposition sans la dépasser, ne fait-on pas que la reconduire $? \gg^{25}$.

N'aurions-nous pas intérêt - au lieu de nous arrêter à «ce que l'homme a sous les yeux » (phrase qui s'inscrit et qui dit un certain rapport de l'occident à la vision), et afin de ne pas rester dans un schéma dichotomique ou manichéen, n'aurions-nous pas intérêt à renouer d'une part avec une lecture telle que celle de Michel Foucault, ou de celle de Georges Bataille ou encore celle de Roland Barthes. Ce dernier ne rappelle-t-il pas à juste titre qu'après avoir dépeint une «construction labyrinthique de l'être» (selon l'expression de G. Bataille), Freud réintroduit une «idéologie du corps humain orienté ${ }^{26}$. Quant à Lacan, dans sa lecture de Freud, il n'a su retenir chez Bataille l'idée d'un mouvement qui ne supporte pas le privilège d'une hiérarchie. Symbole du pouvoir absolu dont la «permanence est souverainement maintenue», l'Obélisque est pour Bataille un monument manifestement phallique. Et Bataille de proposer d'infléchir ce monument en perpétuelle érection.

Cette idée d'un infléchissement et d'un mouvement qui va à l'encontre d'une forme pétrifiée m'incite à aller non pas du côté de l'anthropologie ${ }^{27}$, mais vers un texte. Celui d'Ibn Arabi qui invite également à lire, à déchiffrer,

22. André J., Aux origines féminines de la sexualité, P.U.F., 1995, p. 110.

23. Op. cit., p. 64.

24. Op. cit., pp. 57-58.

25. Levesque C. op. cit., p. 59.

26. Barthes R., «Les sorties du texte», in Bataille, Décade de Cerisy, UGE, 10/18, 1973.

27. M. Schneider se pose cette question : «L'anthropologue doit-il donc s'exiler dans des cultures réputées sauvages pour accéder à des mythes chargés d'un pouvoir analyseur ? ", (Le Paradigme féminin, p. 210). 
à interpréter ou, selon les termes de M. Arkoun, à «transgresser, déplacer, dépasser ».

Philosophe à sa manière, mystique également à sa manière, Ibn Arabi n'en demeure pas moins philologue. Mais la philologie n'est pas sa seule demeure. Sa Somme n'est pas un savoir mais un pluralisé, émietté. Il n'a pas laissé de véritable synthèse. Il déconstuit, coupe, découpe la phrase, introduit ses propres mots pour fissurer un verset ${ }^{28}$. Son écriture ainsi que sa lecture ont cette puissance de fissure. Chez Ibn Arabi, le mot travaille. On peut parler d'une véritable «besogne du mot». Et toutes les ressources du langage sont exploitées : énigmes (ishâra, lattîfa, ce qui est subtil, tellement subtil que le langage ne peut en rendre compte), paradoxes, cassures de la phrase. Ibn Arabi désobjective, désanthropomorphise. Ne considère pas la forme, mais le mouvement. Contre l'immobilité de la forme ou sa pétrification, il prône le mouvement. En tant que philologue, il creuse infatigablement le mot et ne cesse de dégager sa pensée d'un système gouverné par les lois du théologique et de l'organique. Pencher sur son œuvre permet d'un côté de ré-inscrire la sexualité dans un rapport avec le lisible et non le visible, de l'autre d'être vigilant quant à cette logique oppositionnelle qui traverse Généalogie du masculin et Le Paradigme féminin de Monique Schneider qui, en cherchant à rompre avec la primauté accordée par Freud au père et au phallus, se voit prise dans le piège d'une primauté accordée cette foisci à la mère et au vagin. "A la dévagination ou à l'évagination freudienne, écrit Claude Levesque, Monique Schneider oppose une revagination ${ }^{29}$.

Toute autre est la perspective d'Ibn Arabi qui articule la question du masculin et du féminin dans un dépassement de cette logique oppositionnelle en se dégageant de tout psychomorphisme.

Dans son œuvre, masculin et féminin sont des «positions » mobiles, interchangeables, caractérisées par une idée du mouvement. C'est ainsi que nous voyons se dessiner dans les Futûhât ${ }^{30}$ (l'œuvre majeure d'Ibn Arabi), par exemple, la configuration d'une quaternité. Deux couples se dressent : AdamMarie et Eve-Jésus. De même qu'il y eut un féminin existencié d'un masculin sans la médiation d'une mère, de même qu'il y eut un féminin existencié d'un masculin sans la médiation d'un père. En la personne de Marie, le Féminin est investi de la fonction créatrice active. Le rapport de Marie envers Jésus forme l'antitype du rapport d'Eve envers Adam. Jésus et Eve sont frère et sœur tandis que Marie et Adam sont les deux parents. Marie sera identifiée au principe premier $^{31}$.

28. Cf. Abdelouahed H., «Comme si tu Le voyais!», in Art Press. Images et religions du Livre, $\mathrm{n}^{\circ} 25,2004$, pp. 44-47.

29. Levesque C. op. cit., p. 59.

30. Ibn Arabi, Al-Futûhât al-Makkiyya (Les Illuminations de la Mecque), 4 tomes, éd. Dâr Sâdir (sans date d'édition).

31. Or, dans la pensée aristotélicienne, le principe premier est masculin sachant que le masculin est l'homme. 
Si l'on dit à l'instar de M. Foucault que la différence sexuelle ne se donne pas à voir mais à lire, l'exemple du calame (qalam) et de la table (lawh) en fournit une belle illustration, illustration de la mobilité au sein d'une linguistique à définir au sens «guerrier ou encore : érotique», selon la belle expression de Roland Barthes ${ }^{32}$. Le calame dans un mouvement amoureux inscrit, marque, dépose ses traces sur la table. Premier par rapport à la table, il est doté de la fonction active. La table, quant à elle, est réceptive. Elle accepte l'inscription et la reçoit pleinement. Par rapport au calame, elle est seconde. Nonobstant, le calame est-il toujours actif? Est-il toujours premier? Que non! D'un côté, il est Intellect premier, de l'autre, il est rûh (âme, cette dernière étant réceptive et donc seconde). Et le Lisân al- 'arab de définir le calame comme mabtûr, amputé (ce qui a subi une amputation, séparé d'un reste qui choit). Toutefois, ce calame n'est pas une chose et n'a pas d'objectivité. Il n'est pas objectivable. Il est entre autres un attribut que peut s'approprier celui qui parvient à se doter des qualités du calame. Dans le lexique akbarien (celui d'Ibn Arabi), il a pour synonyme la table (lawh) qui est active par rapport à ce qui lui est postérieur, réceptive par rapport à ce qui lui est antérieur. Wahâkadhâ kullu fâ'il wa munfail: lawh wa qalam (il en va ainsi de chaque actif et de chaque passif: chacun est-il table et calame). Ainsi, la réalité du féminin et la réalité du masculin (haqîqat al-unûtha wa haqîqat adhdhukûra circulentelles dans toute créature ${ }^{33}$. Chaque créé rassemble en lui ces deux réalités : la réalité féminine et la réalité masculine.

Ceci nous invite à reprendre un thème qui revient comme un leitmotiv dans l'écrit de Monique Schneider, à savoir cette dichotomie introduite par Freud entre le père qui bénéficie des hauteurs célestes de l'intelligible et la mère à qui échoit le domaine du sensible et de la sensorialité. Ibn Arabi considère différemment la paternité et la maternité. L'exemple de l'architecte et du menuisier en est une illustration. La parole de l'architecte, dit-il, est père, le qubûl, la faculté réceptive (le fait d'accepter de recevoir) chez celui qui entend est mère. La trace est un enfant. L'architecte n'est pas père pas plus que le menuisier n'est une mère. C'est le matériau sonore du mot prononcé qui est père, c'est la faculté réceptive du matériau sonore qui est mère. La trace - le monde pour Ibn Arabi étant un monde de désir - qui naît de cette rencontre est un enfant. Quant à la verticalité, thème récurrent de la Généalogie, pour Ibn Arabi, elle n'est nullement celle d'un organe, mais plutôt du souffle ou de l'air, l'air de phôné qui exerce une action motrice traçant l'image d'un mot qui sera capté dans sa toute sensorialité et sa capacité d'engendrement inhérente à l'acte de dire. Ainsi, l'opération de l'esprit comme processus civilisateur n'est pas en rupture avec la sensorialité. La verticalité n'est pas de l'ordre de l'anthropomorphisme. Elle est celle

32. Barthes R., op. cit., p. 61 (Tant il est vrai qu'en arabe le tibâq (concordance des deux sexes opposés) et le jinâs (la rencontre au sens érotique) sont deux figures de style).

33. Cf. entre autres Fut. I., p. 139; Fut. I., p. 148; 'Uqlat al-Mustawfiz, pp. 55-56; Al-Hakîm S., Al-Mu’jam al-Sûfì, éd. Dandara, Beyrouth, 1981. 
du souffle disant les lettres. Et le redressement de l'homme ou son éloignement du sol (lever le nez $=$ faire le dédaigneux ${ }^{34}$ ) devient mouvement de la langue se dégageant de la littéralité du sol pour la métaphore. L'éloignement du sol devient écart s'instituant au cœur même de l'opération langagière, le mot pouvant ainsi s'accorder la «sensation verticale de sa possible figure (Pierre Fédida) $^{35}$.

L'obélisque de la Concorde, dit Bataille, devrait se pencher, quitter cette position de l'érection immobile. Or, dans la science des lettres ${ }^{36}$, le alif (1'Alef), sous la puissance d'éros, consent à se pencher (yanhanî de la même racine que hanân, tendresse) afin d'enlacer, épouser une autre lettre (yamîl, écrit Ibn Arabi, avoir un penchant pour l'autre). Quand l'alif(l'Alef) parvient à enlacer tendrement ou amoureusement le lâm (le «l»), ils forment le signe sémantique de la négation en arabe. Laquelle négation est abordée par Monique Schneider de la façon suivante: «n'être pas la femme ou n'être pas la mère ». Là, dit Monique Schneider «On est amené à prendre acte de la présence du féminin au sein de l'opération qui dit l'éloignement à l'égard de ce même féminin » ${ }^{37}$. La décision qui, selon Freud, fonde la civilisation correspond à une «évagination », continue Monique Schneider, à un rejet du féminin (du vagin) et de la mère. Monique Schneider rappelle que dans la langue allemande etscheiden signifie décider, juger, trancher et scheide désigne le vagin. En arabe, le jugement se dit ra'y et la femme, mar'a. Les deux termes non seulement ne s'excluent pas mais partagent la même racine ou la même demeure.

Si l'on suit cette idée que le sexe est plutôt à lire, à interpréter, à déchiffrer (qara'a d'ailleurs en arabe signifie aussi bien lire, le mouvement de lecture, que cette opération par laquelle la femelle retient la substance du mâle, l'accent étant mis sur la réceptivité de la femelle), que le sexe n'est pas dans le visible, n'aurions-nous pas là l'un des sens de cette phrase de Tabarî qui insiste sur le lien entre la vue et le génital et stipulant que le sexe n'est lié à la vision que par l'interdit? Mais on pourrait aller plus loin si l'on dit que le farj impliquant l'idée de fente, d'interstice et d'ouverture, appelle également cet interstice où tombe justement tout savoir sur le sexe. Faraja fâhu, fatahahu lilmawth, écrit Ibn Manzûr (XII-XIII siècle), faraja sa bouche signifie «il l'a ouverte pour la mort» le Maître absolu. Lacan avait raison d'écrire que le langage « ne domine pas ce fondement du sexe», ce dernier étant «relié à l'essence de la mort» ${ }^{38}$. Et l'une des multiples significations du farj est justement la limite. L'on comprend également cette définition du farj comme ce qui est mukhîf, inquiétant. Et si l'on se souvient qu'al-

34. Freud S., Naissance de la psychanalyse, «Lettre 75 », P.U.F., p. 205.

35. Cette question fut traitée in Abdelouahed H., « Les mots qui peignent ou la garantie de la ressemblance », in La Vie sensorielle, P.U.F., 2002, pp. 109-127.

36. La science des lettres est la science qui traite chez les Arabes de l'horizontalité et de la verticalité au sein du langage.

37. Schneider M., Généalogie du masculin, p. 339.

38. Lacan J., La Logique du fantasme, 1967, inédit (Séance du 18 janvier). 
'awra (autre mot pour désigner le farj) signifie «ce qui est borgne», et que cette 'awra est «le lieu du secret divin» ${ }^{39}$, l'on ne peut que faire nôtre cette maxime: «Le secret du sexe est du côté du Réel». Ainsi, si al- 'awra est le lieu du secret divin, c'est dans le hors langage que se loge le secret du sexe.

Le masculin et le féminin ne sont pas des positions, car position comme le dit justement Monique Schneider renvoie à la corporéité d'une immobilité ne serait-ce que provisoire. Or, le masculin et le féminin circulent (yasriyân). Et cette idée de sarayân (circulation) revient sous la plume d'Ibn Arabi, au sujet du Nom du Père ${ }^{40}$, de la verticalité ${ }^{41}$ et du langage. Il écrit justement à ce sujet : «c'est par ce qu'il circule qu'il nécessite un ridâ', manteau » ${ }^{42}$. Or, lorsque Freud prend l'exemple de l'image onirique du manteau, écrit Monique Schneider, celle-ci se trouve dynamitée par « l'effet du signifiant ». En allemand, manteau se dit Mantel, terme dont Freud souligne que la première syllabe est homophone de Mann, homme ${ }^{43}$. Or, lorsqu'il rappelle que le ridâ' (manteau) est l'homme (au-delà de l'être humain, le sujet de la parole), Ibn Arabi attire l'attention sur le fait que ridâ' est de la même racine que radâ (la mort).

La question qui est en train de se dessiner - et qui restera ouverte pour le moment - porte sur le rapport de l'inconscient à l'homophonie ou à l'étymologie et le lien privilégié que tisse ou entretient une langue avec l'une ou l'autre.

Même s'il parle d'un masculin et d'un féminin qui circulent, Ibn Arabi insiste sur la féminité qui circule, al-unûtha sâriya fìl-wujûd, écrit-il. Est-ce la féminité originaire refoulée, celle de l'enfant effractée ? Refoulée se dit, en arabe, makbûta. On peut même se représenter cette métaphore laplanchienne, d'un dédale empêchant le bas de remonter à la surface ${ }^{44}$. Trahirais-je sa pensée si je disais que au-delà du masculin et du féminin, il y a une féminité qui circule ? Féminité dont les procédés du discours en rendent compte. Ibn Arabi rappelle le hadîth (parole de prophète Muhammad) : «Trois choses de votre monde me furent rendues dignes d'amour : les femmes, le parfum et la prière ». Ce qui est intéressant et inhabituel c'est que dans ce hadîth, le féminin l'emporte sur le masculin ${ }^{45}$. Le conformisme grammatical subit ici une altération. Ibn Arabi

39. Fut. I., p. 680.

40. Fut. I., p. 138 ; Fut. IV., p. 298.

41. Fut. IV., pp. 291-292.

42. Fut. II., p. 104.

43. M. Schneider, Le Paradigme féminin, p. 311.

44. Jean Laplanche donne cette métaphore dans sa définition du refoulement originaire. Cf. Problématiques IV. L'inconscient et le ça, P.U.F., 1981.

45. Le prophète dit: «thalâthun». Or, «thalâthun» désigne trois choses féminines. Pour parler de trois choses masculines ou de deux choses féminines et une chose masculine, nous disons : «thalâthatun ». Dans ce hadîth où le prophète nomme les femmes, la prière (terme également féminin en arabe) et le parfum ( $a l$-tîb, terme masculin), il fallait, de point de vue grammatical, que le masculin l'emportât sur le féminin. Muhammad faillit donc à la règle grammaticale puisque dans ce hadîth, le féminin l'emporte sur le masculin. 
s'empare de cet exemple linguistique pour attester une réalité métaphysique, disait Henry Corbin, à savoir que tous les termes qui marquent l'origine et la cause sont des termes féminins et que si la phrase est incorrecte du point de vue grammatical, elle suggère néanmoins que le féminin est l'origine de toute chose. Dès qu'on spécule sur l'origine et la cause, on constate, dit Ibn Arabi, cette antériorité du féminin ${ }^{46}$. Nous souscrivons à la phrase d'H. Corbin à condition toutefois de ne pas oublier la nécessité - dans le champ qui est le nôtre - de traduire la métaphysique en métapsychologie.

C'est ainsi que nous faisons remarquer que la féminité est constamment présente dans l'œuvre d'Ibn Arabi et que la jeune fille, cette princesse $\min$ banât al-rûm, «d'entre les filles des Grecs », selon la traduction de H. Corbin, n'est pas tant une figure déterminée - comme on a pu l'écrire - que la métaphore d'une féminité dans sa marche interprétative et son ouverture sur l'ailleurs ${ }^{47}$.

A la lumière de ce qui précède, ce n'est pas tant la revagination qui nous intéresse que l'inscription d'une féminité comme ouverture définissant le sujet de la parole. Le vagin, organe féminin, se dit mahbal en arabe, à savoir le lieu de la folie. Or, al-farj est le sexe aussi bien de l'homme que de la femme. Et le Lisân al 'arab d'insister: c'est al- 'awra lirrijâl wa li nisâ' (c'est al- 'awra, ce qui est frappé par la cécité, pour les hommes et pour les femmes). C'est aussi al-bâriz al-munkachif al-zâhir (l'apparent) wa kadhâlika al-unthâ (ceci est également pour la femme). Il s'agit de ce qui apparaît comme fente, ouverture. Al-unthayân (les deux féminines) sont les testicules.

Pierre Fédida soulève cette question de l'organe emblématique et souligne que dans Pour introduire le narcissisme, Freud parle de la modification, sous l'effet de la libido, de l'organe ${ }^{48}$. Ce qui compte, écrit Pierre Fédida, c'est sa capacité d'être modifié. Toutefois, si l'on se souvient du alif qui, mû par le désir, se penche pour épouser le lâm, l'on peut dire à l'instar de Jean-Luc Nancy qu'il ne s'agit pas tant de la modification que de l'ouverture sur l'autre. L'alternative, écrit Jean-Luc Nancy n'est pas celle de l'érection de soi et de la rétraction, mais celle de l'ouverture et de la fermeture.

C'est parce qu'il y a ouverture qu'il y a un rapport à l'ailleurs.

Dans ce rapport à l'ailleurs, quand le nom et la chose aspiraient à l'union et que l'un disait à l'autre «donne-moi cela», la seule réponse qui pouvait se

46. Ibn Arabi, Fusûs al-hikam. Ed. Dâr al-Kitâb al-'arabî, Beyrouth, 1946, p. 220 ; trad. Burckardt T., La Sagesse des prophètes, Albin Michel, 1974, p. 207.

47. Dans Turjumân al-ashwâq (L'Interprète des ardents désirs), c'est elle qui invite la pensée du poète à s'ouvrir sur un ailleurs.

48. « Eh bien, nous connaissons le modèle d'un organe douloureusement sensible, modifié en quelque façon sans être pourtant malade, au sens habituel : c'est l'organe génital en cas d'excitation. Il est alors congestionné, turgescent, humide, et le siège de sensations diverses. » Freud S., «Pour introduire le narcissisme », in La Vie sexuelle, P.U.F., 1969, p. 90. 
dessiner: «comment te donner ce que je n'ai pas ?» ${ }^{49}$. Donner ce que l'on n'a pas. Blanchot disait: il n'est pas d'autre don.

Si la tâche du traducteur consiste à racheter dans sa propre langue «ce pur langage exilé dans la langue étrangère ${ }^{50}$, c'est à partir de la perte, de l'exil dans l'autre langue, l'ouverture sur ce qui est étreint et pourtant ignoré qu'un travail de pensée peut être possible. L'écho de l'original porte sur une ouverture. Cette ouverture qui définit la sexualité de tout un chacun est ce qui conditionne la possibilité même de traduction.

\author{
Houriya ABDELOUAHED \\ 83 rue Napoléon Fauveau \\ 95170 Deuil-la-Barre
}

\title{
BIBLIOGRAPHIE
}

ABDELOUAHED H. « Abraham entre doute et certitude », in Revue internationale de psychopathologie, $\mathrm{n}^{\circ} 16,1994$, pp. 579-590.

ABDELOUAHED H., « Des mots qui peignent ou la garantie de la ressemblance », in $\mathrm{La}$ Vie sensorielle, P.U.F., 2002, pp. 109-127.

ABDELOUAHED H., «Comme si tu Le voyais ! », in Artpress. Images et religions du Livre, $\mathrm{n}^{\circ} 25,2004$, pp. 44-47.

AL-HAKÎM S., Al-Mu'jam al-sûfi, éd. Dandara, Beyrouth, 1981.

ANDRÉ J., Aux origines féminines de la sexualité, P.U.F., 1995.

BARTHES R., «Les sorties du texte », in Bataille, Décade de Cerisy, UGE, 10/18, 1973.

BENSLAMA F., «Le sexe absolu », in Cahiers intersignes, ${ }^{\circ} 2$, printemps 1991, pp. 105124.

BENJAMIN W., Mythe et violence, I, Denoël, 1971.

FÉDIDA P., Par où commence le corps humain, P.U.F., 2000.

FOUCAULT M., Histoire de la sexualité, I, Gallimard, 1976.

FREUD S., «Pour introduire le narcissisme», in La Vie sexuelle, P.U.F., 1969, pp. 81-105.

GOMEZ MANGO E., La Mort enfant, Gallimard, coll. «Tracé», 2003.

HÉRITIER F., Masculin/féminin, éd. Odile Jacob, 1996.

IBN ARABI, Al-Futûhât al-Makkiyya (Les Illuminations de la Mecque), 4 tomes, éd. Dâr Sâdir (sans date d'édition).

IBN ARABI, Fusûs al-Hikam, Beyrouth, éd. Dâr al-Kitâb al'- ‘arabî, 1946 ; trad. Burckardt T., La Sagesse des prophètes, Albin Michel, 1974.

49. Sur la rencontre entre le nom et la chose, $c f$. Fut. I., pp. 100-101. Pour la traduction en français de ce chapitre, $c f$. «Les noms et les choses. La rencontre qui articule le monde. Traduction et commentaire », in Cliniques méditerranéennes, $\mathrm{n}^{\circ}$ 68, 2003, pp. 109-122.

50. Walter Benjamin, op.cit., p. 273. 
IBN ARABI, Turjumân al-Ashwâq, éd. Dâr Sâdir, (sans date d'édition). LACAN J., Le Séminaire, Livre III, Les psychoses, Seuil, 1981.

LACAN J., La Logique du fantasme, 1967, inédit.

LAPLANCHE, Problématiques IV. L'inconscient et le ça, P.U.F., 1981.

LEVESQUE C., Par-delà le masculin et le féminin, Aubier, 2002.

SCHNEIDER M., Généalogie du masculin, Aubier, 2000.

SCHNEIDER M., Le Paradigme féminin, Aubier, 2004.

SCHUR M., La Mort dans la vie de Freud, Gallimard, 1975.

TABAR̂̂, Jâmi' al-bayân 'an tafsîr âyi al-Qur'ân, Beyrouth (Liban), éd. Dâr al-fikr, 1988.

\section{Houriya Abdelouahed - La féminité et le mouvement}

Résumé : La définition par Lisân al- 'arab (encyclopédie philologique de la langue arabe) du mot farj comme ouverture, fissure, interstice... permet non seulement de mesurer l'écart entre l'original et ce que peut être une traduction, mais de réfléchir, d'un côté, sur ce que Freud et Lacan nomment «le primat du phallus», de l'autre sur une féminité originaire dont la langue rend compte. Cette féminité originaire comme ouverture est ce qui définit la sexualité de tout un chacun et qui conditionne la possibilité même de traduction.

Mots-clés : Sexe - Ouverture - Féminité - Phallus - Masculin/féminin - Langue Traduction.

\section{Houriya Abdelouahed - Femininity and Movement}

Summary : The definition of the word farj in the Lisân al- 'arab (Philological Encyclopaedia of Arabic) as an opening, a gash or hole... not only allows us to take full stock of the gap between the original and its translation, but also leads us to muse firstly on what Freud and Lacan term as the 'primacy of the phallus' and secondly on the notion of originary femininity that language takes into account here. This originary femininity as defined here as an opening is what defines the sexuality of each and every one of us and also makes the act of translating itself possible.

Key-words : Sex - Opening - Femininity - Phallus - Masculine/Feminine - Language - Translation. 